PROCEEDINGS OF THE

AMERICAN MATHEMATICAL SOCIETY

Volume 133, Number 12, Pages 3619-3626

S 0002-9939(05)08026-3

Article electronically published on June 8, 2005

\title{
GEOMETRIC CRITERIA FOR THE NONEXISTENCE OF CYCLES IN GAUSE-TYPE PREDATOR-PREY SYSTEMS
}

\author{
YAPING LIU \\ (Communicated by Carmen C. Chicone)
}

\begin{abstract}
The global stability of a multi-species interacting system has apparently important biological implications. In this paper we study the global stability of Gause-type predator-prey models by providing new criteria for the nonexistence of cycles and limit cycles. Our criteria have clear geometrical interpretations and are easier to apply than other methods employed in recent studies. Using these criteria and related techniques we are able to develop new results on the existence and uniqueness of cycles in Gause-type models with various growth and response functions.
\end{abstract}

\section{INTRODUCTION}

Since the seminal work of Kolmogorov [9], extensive work has been done on the existence and uniqueness of limit cycles in a predator-prey system modeled by two autonomous differential equations. One very popular version of such a system, the so-called Gause-type model, has the following general form:

$$
\left\{\begin{array}{l}
\frac{d x}{d t}=x g(x)-y p(x), \\
\frac{d y}{d t}=y[c p(x)-d] .
\end{array}\right.
$$

Here $c$ and $d$ are positive real numbers, $g(x)$ is the individual growth rate of the prey species in the absence of the predators, and $p(x)$ represents the functional response of predators to the growth of prey. A prototype of $g(x)$ is the logistic growth pattern, while $p(x)$ is usually assumed to be monotonically increasing. For the background on this model and its generalizations, see Freedman [4, p. 66-81], Kuang and Freedman [12, Huang and Merrill [8], and the references therein.

Motivated by biological applications, one focus of study about a predator-prey system is the global stability of the system in the positive cone of the phase plane $\{(x, y) \mid x>0, y>0\}$. A solution $(x(t), y(t))$ of system (1) is said to be positive if $x(t)>0$ and $y(t)>0$ for all $t \geq 0$. The global stability of the system is typically determined by the existence of a positive attractor, either an equilibrium or a limit cycle. For this reason, the existence and uniqueness of positive cycles and limit cycles of system (1) has attracted much interest in recent years. For a sample of

Received by the editors August 13, 2004.

2000 Mathematics Subject Classification. Primary 34D23; Secondary 34C07, 92D25.

Key words and phrases. Geometric criterion, global stability, limit cycle, predator-prey system, Dulac's theorem, Liénard system.

(C)2005 American Mathematical Society Reverts to public domain 28 years from publication 
these studies, see Cheng [1, Cheng et al. 2], Kuang and Freedman [12, Huang and Merrill [8], Ding [3, Kuang [11, Kooij and Zegeling [10, Sugie et al. [19], Sugie [17, Moghadas [14, and Xiao and Zhang [21]. Most of the recent work has employed technical methods, such as transforming the system to an equivalent generalized Liénard system or trying directly to exploit the special structure of the limit cycle and the prey isocline. Because of the complexity involved, geometrical interpretation of the results in these studies were rarely provided, and therefore the applicability and the biological implication of their results are not easily perceived.

In the next two sections, we will study the global stability of the system by presenting a few geometric criteria for the nonexistence of cycles. Our criteria have clear geometrical interpretations and are easier to apply than most other methods. In Sections 4, 5, and 6, we apply these criteria to a few different models to illustrate their wide applicability. Using our methods one can reproduce many recent results. More importantly, new results can be developed on the existence and uniqueness of cycles and limit cycles in models with various types of growth and response functions.

\section{Geometric Criteria}

Throughout this paper, by a cycle we mean a nontrivial positive cycle. The following hypotheses on $g(x)$ and $p(x)$ in model (1) are typical:

(H1) $g(x) \in C^{3}[0,+\infty)$, and there exists a $K>0$ such that $(x-K) g(x)<0$ for $x \geq 0, x \neq K$.

(H2) $p(x) \in C^{3}(0,+\infty), p(0)=0, p^{\prime}(x)>0$ for $x>0$, and $c p(K)>d$.

In (H1) the value $K$ is interpreted as the carrying capacity of the prey species in the absence of the predators. In (H2) we do not require the smoothness of $p(x)$ at $x=0$, because in some models it may happen that $p^{\prime}\left(0^{+}\right):=\lim _{x \rightarrow 0^{+}} p^{\prime}(x)=+\infty$ (see the examples in later sections). On the other hand, we require $c p(K)>d$ in (H2) because the global stability of the system with $c p(K) \leq d$ is trivial, and is not considered here.

It follows from (H2) that there is a unique positive $x^{*}<K$ such that $c p\left(x^{*}\right)=d$. One unique feature of Gause-type models is that the predator isocline in the positive cone, i.e., the curve determined by the equation $c p(x)-d=0$, is a vertical line $x=x^{*}$. Meanwhile, the prey isocline is given by the function $F(x):=x g(x) / p(x)$. The unique intersection point $\left(x^{*}, y^{*}\right)$ of the isoclines, with $y^{*}=F\left(x^{*}\right)$, is the only positive equilibrium of system (1). For convenience, we will fix the notation $R(x)=1 / p(x)$ and $\phi(x)=F^{\prime \prime}(x) / R^{\prime}(x)$ for the rest of this paper. The first main result is

Theorem 1. Let (H1) and (H2) be satisfied. Assume that $\phi(x)$ satisfies the condition

$$
\left(x-x^{*}\right)\left[\phi(x)-\phi\left(x^{*}\right)\right]>0
$$

for $0<x<K, x \neq x^{*}$. Then system (1) has no cycle if and only if $F^{\prime}\left(x^{*}\right) \leq 0$.

Proof. Using linear stability analysis (Jacobian) and phase plane analysis it is easy to see that the only two boundary equilibria of the system, $(0,0)$ and $(K, 0)$, are always saddle points, while $\left(x^{*}, y^{*}\right)$ can be a stable or unstable focus or node depending on whether it lies on an upslope or a downslope of $F(x)$. For $F^{\prime}\left(x^{*}\right)>0$, $\left(x^{*}, y^{*}\right)$ is a repeller. It is known that under the hypotheses (H1) and (H2), the positive solutions of (1) are eventually uniformly bounded. The existence of a limit 
cycle then results from the Poincaré-Bendixson Theorem. The proof is standard and the details are omitted.

To show the nonexistence of cycles when $F^{\prime}\left(x^{*}\right) \leq 0$, we apply Dulac's theorem. Define the Dulac function by $D(x, y)=R(x) y^{\alpha-1}$, where $\alpha$ is a real number that is to be determined. The divergence of the vector field $(D d x / d t, D d y / d t)$ is $y^{\alpha-1} G(\alpha, x)$, where $G(\alpha, x)=F^{\prime}(x)+\alpha(c-d R(x))$. From phase plane analysis and the fact that a cycle of a $C^{1}$ system must contain an equilibrium in its interior, we see that all cycles of (1) lie in the strip $S=\{(x, y) \mid 0<x<K, 0<y<+\infty\}$. To apply Dulac's theorem in this strip, we would like to show that for some appropriately chosen $\alpha, G(\alpha, x) \leq 0$ for all $x \in(0, K)$ and $G(\alpha, x)$ is not identically zero on any interval of $x$.

Define $\alpha^{*}=\frac{F^{\prime \prime}\left(x^{*}\right)}{d R^{\prime}\left(x^{*}\right)}$. Then $G_{x}\left(\alpha^{*}, x\right)=F^{\prime \prime}(x)-\alpha^{*} d R^{\prime}(x)=R^{\prime}(x)\left[\phi(x)-\phi\left(x^{*}\right)\right]$ and $G_{x}\left(\alpha^{*}, x^{*}\right)=0$. Using the fact that $R^{\prime}(x)=-p^{\prime}(x) /[p(x)]^{2}<0$ for $x>0$, we have $G_{x}\left(\alpha^{*}, x\right)>0$ for $x<x^{*}$ and $G_{x}\left(\alpha^{*}, x\right)<0$ for $x>x^{*}$. So $G\left(\alpha^{*}, x^{*}\right)=F^{\prime}\left(x^{*}\right)$ is a global maximum of $G\left(\alpha^{*}, x\right)$.

It follows from the simple assumption on $\phi(x)$ in Theorem 1 that local asymptotic stability of $\left(x^{*}, y^{*}\right)$ implies its global asymptotic stability. Different configurations of $\phi(x)$ can be proposed to satisfy the condition in Theorem 1. The simplest one is the following

Corollary 1. Let (H1) and $(\mathrm{H} 2)$ be satisfied. Let $\phi(x)$ be an increasing function on $(0, K)$. Then system (1) has no cycle if and only if $F^{\prime}\left(x^{*}\right) \leq 0$.

Corollary 2. Let (H1) and (H2) be satisfied. If $F(x)$ strictly decreases for $0<$ $x<K$, then system (1) has no cycle.

Proof. This can be obtained by taking $\alpha=0$ in the proof of Theorem 1 . It is also a corollary of Theorem 3.2 in Hsu [7. Also see Theorems 3.2 and 4.2 in Kuang [11.

The idea of using the parameter $\alpha$ in applying Dulac's theorem in the proof of Theorem 1 is of course not new. See for example, Hsu 7], Hofbauer and So 6], Kuang [11, and Ruan and Xiao [16]. But our improvement of the method is in choosing an appropriate formula for $\alpha$. In fact, the choice of $\alpha^{*}=\frac{F^{\prime \prime}\left(x^{*}\right)}{d R^{\prime}\left(x^{*}\right)}$ is derived from the solution of a minimax problem and therefore is usually optimal. To illustrate this idea, we make the following assumption:

(H3) There exists an interval $I_{0}=\left(a_{0}, b_{0}\right)$ containing $\alpha^{*}$ such that for any $\alpha \in I_{0}$, $F^{\prime \prime \prime}(x)-\alpha d R^{\prime \prime}(x)<0$ for $x>0$.

The idea regarding (H3) will become clearer later. We remark that in many popular models (H3) is satisfied.

Proposition 1. Let (H1) through (H3) be satisfied. Then the choice of $\alpha^{*}=$ $\frac{F^{\prime \prime}\left(x^{*}\right)}{d R^{\prime}\left(x^{*}\right)}$ used in the proof of Theorem 1 is optimal in the sense that $\max _{0<x<+\infty} G(\alpha, x)$ is minimized at $\alpha^{*}$ for $\alpha$ in some maximal interval $I_{m}$.

Proof. Recall $G(\alpha, x)=F^{\prime}(x)+\alpha(c-d R(x))$. Let $H(\alpha, x)=G_{x}(\alpha, x)=F^{\prime \prime}(x)-$ $\alpha d R^{\prime}(x)$. We have $H\left(\alpha^{*}, x^{*}\right)=0$ and $H_{x}(\alpha, x)<0$ for all $\alpha \in I_{0}$ and $x>0$. By the Implicit Function Theorem, the equation $H(\alpha, x)=0$ defines $x=x(\alpha)$ as a unique function of $\alpha$ in a neighborhood of $\alpha^{*}$. It follows from $H_{\alpha}=-d R^{\prime}(x)>0$ that $\frac{d x(\alpha)}{d \alpha}=-\frac{H_{\alpha}}{H_{x}}>0$. So $x(\alpha)$ is an increasing function of $\alpha$. 
Let $I_{m}=\left(a_{m}, b_{m}\right)$ be the maximal interval containing $\alpha^{*}$ such that the implicit function $x(\alpha)$ can be extended. We claim that $I_{m}$ contains all $\alpha \in I_{0}$ such that the equation $H(\alpha, x)=0$ has a solution $x>0$. Assume on the contrary that there exists some $\alpha_{1} \in I_{0} \backslash I_{m}$ and some $x_{1}>0$ such that $H\left(\alpha_{1}, x_{1}\right)=0$. Without loss of generality, let $\alpha_{1}<a_{m}$. Choose any $\alpha_{2} \in I_{m}$ and let $x_{2}=x\left(\alpha_{2}\right)$. Then $x_{1}<x_{2}$. For any $\alpha \in\left(\alpha_{1}, \alpha_{2}\right)$, we have $H\left(\alpha, x_{1}\right)>H\left(\alpha_{1}, x_{1}\right)=0$, and $H\left(\alpha, x_{2}\right)<H\left(\alpha_{2}, x_{2}\right)=$ 0 . The Intermediate Value Theorem then implies that an $x_{\alpha} \in\left(x_{1}, x_{2}\right)$ exists such that $H\left(\alpha, x_{\alpha}\right)=0$. This means $I_{m}$ can be extended below $a_{m}$, a contradiction.

The system of equations $G_{x}=F^{\prime \prime}(x)-\alpha d R^{\prime}(x)=0$ and $G_{\alpha}=c-d R(x)=0$ gives the unique critical point $\left(\alpha^{*}, x^{*}\right)$ of the function $G(\alpha, x)$. By using the Hessian matrix of $G$ we can see that this critical point is a saddle. More explicitly, for any given $\alpha \in I_{m}, G(\alpha, x)$ has a global maximum at $x(\alpha)$ for $x>0$. Setting $f(\alpha)=G(\alpha, x(\alpha))$ for $\alpha \in I_{m}$, we see that

$$
f^{\prime}(\alpha)=G_{\alpha}+G_{x} \frac{d x(\alpha)}{d \alpha}=c-d R(x(\alpha))+H(\alpha, x(\alpha)) \frac{d x(\alpha)}{d \alpha}=c-d R(x(\alpha)) .
$$

Thus $f^{\prime}\left(\alpha^{*}\right)=c-d R\left(x^{*}\right)=0$ and $f^{\prime \prime}(\alpha)=-d R^{\prime}(x(\alpha)) \frac{d x(\alpha)}{d \alpha}>0$, so $f\left(\alpha^{*}\right)$ gives the global minimum for $\alpha \in I_{m}$, as we desired.

Corollary 3. Let (H1), (H2) and (H3) be satisfied. Then system (1) has no cycle if and only if $F^{\prime}\left(x^{*}\right) \leq 0$.

\section{Geometrical interpretation}

To get a better idea on when the above results are applicable, we will restrict our attention to the following three typical types of the prey isocline:

Type 1. $F(x)$ decreases for $0<x<K$. Either $F^{\prime \prime}(x)<0$ for $x>0$ or $F^{\prime \prime}(x)$ changes sign at some $\tilde{x}>0$. See Figure 1(a). Following Corollary 2, this case is trivial.

Type 2. $F(x)$ has a unique maximum at some $x_{M}>0$ and no interior minimum for $x>0$. Again either $F^{\prime \prime}(x)<0$ for $x>0$ or $F^{\prime \prime}(x)$ changes sign at some $\tilde{x}>0$. See Figure 1(b).

Type 3. $F(x)$ has a unique local minimum at $x_{m}$ and a unique local maximum at $x_{M}$ with $0<x_{m}<x_{M}<K$. The graph of $F(x)$ has two downslopes, one for $0<x<x_{m}$ and the other for $x>x_{M}$. There is an $\tilde{x} \in\left(x_{m}, x_{M}\right)$ such that $(x-\tilde{x}) F^{\prime \prime}(x)<0$ for $x \neq \tilde{x}$. See Figure $1(\mathrm{c})$.

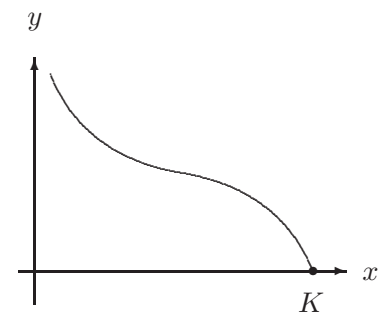

(a) Type 1

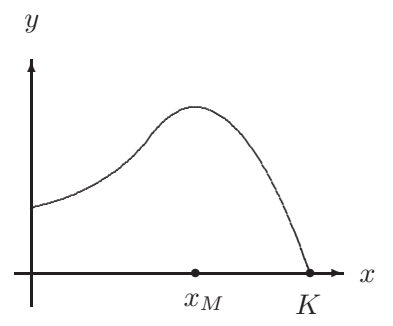

(b) Type 2

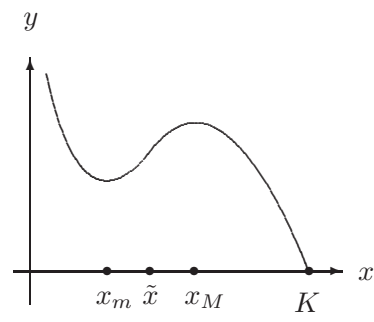

(c) Type 3

Figure 1. The three types of the prey isocline

One can check with relative ease that in basically all important models proposed in practice, $F(x)$ is one of these three types. Similar classification of the prey 
isocline has been given in Freedman [4, p. 74] and Wolkowicz [20, both having one important exclusion: $F\left(0^{+}\right)=+\infty$ for a type 1 or a type 3 function, where $F\left(0^{+}\right)$ is defined to be $\lim _{x \rightarrow 0^{+}} F(x)$.

Note that $G(\alpha, x)=F^{\prime}(x)-\psi(x)$, where $\psi(x)=\alpha[d R(x)-c]$. Geometrically, for Dulac's theorem to work we need to choose a real number $\alpha$ so that the curve $\psi(x)$ lies totally above the curve $F^{\prime}(x)$. Disregarding the trivial case when $F(x)$ is of type 1 , there are three cases in which Theorem 1 applies: (a) when $\left(x^{*}, y^{*}\right)$ is on the only downslope of a type 2 function $F(x)$, (b) when $\left(x^{*}, y^{*}\right)$ is on the right downslope of a type 3 function $F(x)$, and (c) when $\left(x^{*}, y^{*}\right)$ is on the left downslope of a type 3 function $F(x)$. This is illustrated in Figure 2.

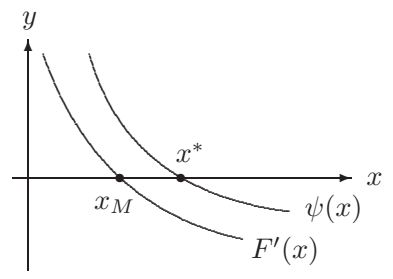

(a) Type $2, F^{\prime \prime}\left(x^{*}\right)<0$

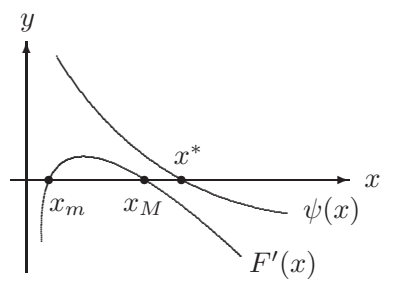

(b) Type $3, F^{\prime \prime}\left(x^{*}\right)<0$

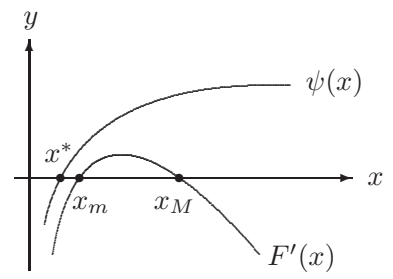

(c) Type $3, F^{\prime \prime}\left(x^{*}\right)>0$

Figure 2. Three cases in which Theorem 1 applies

Different criteria have been proposed by a few authors for the global stability of $\left(x^{*}, y^{*}\right)$. Hsu 7 claimed that if the prey isocline $F(x)$ is concave down, then when the positive equilibrium $\left(x^{*}, y^{*}\right)$ is on the downslope of $F(x)$, cycles do not exist. Unfortunately this simple geometric criterion was shown to be wrong. A concrete counterexample was constructed in Hofbauer and So [6]. To different degrees Cheng et al. [2, Kuang [11], Moghadas [14], and Xiao and Zhang [21] contain modifications of Hsu's result. But the sufficient conditions they proposed are difficult to satisfy and hard to verify, and the geometrical interpretation of their results is not clear.

Our results above show that the concavity assumption on the prey isocline can actually be weakened so that the criteria apply to a wider class of models. In particular, they allow us to deal with models in which $F(x)$ is concave up for small $x>0$. On the other hand, we bring $F^{\prime \prime \prime}(x)$ and $R^{\prime \prime}(x)$ into the picture to make the conditions sufficient.

\section{INVERTED IVLEV'S RESPONSE FUNCTION}

In this section and the next we will study the existence and uniqueness of cycles in two particular models. First we introduce the following uniqueness result.

Lemma 1. If for all real values $\alpha$ the function $F^{\prime}(x)+\alpha(c-d R(x))$ has no positive multiple root, then system (1) has at most one limit cycle which is hyperbolic if it exists.

Proof. The idea is to transform system (1) to an equivalent generalized Liénard system. The following substitutions are standard: replacing $t$ by $-t / p(x)$ and letting $y=e^{Y}$. Then

$$
\frac{d x}{d t}=-\xi(Y)-F(x), \quad \frac{d Y}{d t}=\eta(x),
$$


where $\xi(Y)=-e^{Y}, F(x)=x g(x) / p(x)$, and $\eta(x)=d R(x)-c$ with $R(x)=1 / p(x)$. The result then follows from the corresponding uniqueness results for generalized Liénard systems. See, for example, Kooij and Zegeling [10].

Now consider the response function defined by $p(x)=e^{-a / x}$ with $a>0$ and $p(0):=0$. We name $p(x)$ the inverted Ivlev's response function because it is obtained by inverting the growth rates of Ivlev's function $1-e^{-a x}$ at 0 and $+\infty$.

Using the prototype logistic growth function $g(x)=r(K-x)$ with $r, K>0$, we have $F(x)=r x(K-x) e^{a / x}$. Check that if $K \leq(3+2 \sqrt{2}) a$, then $F^{\prime}(x) \leq 0$ on $(0, K)$ and $F(x)$ is of type 1 . Thus we require $K>(3+2 \sqrt{2}) a$. Then $F(x)$ is of type 3 .

Theorem 2. Let $g(x)$ and $p(x)$ be as given above. Then system (1) has no cycle if $F^{\prime}\left(x^{*}\right) \leq 0$. It has a unique stable limit cycle if $F^{\prime}\left(x^{*}\right)>0$.

Proof. First let $F^{\prime}\left(x^{*}\right) \leq 0$. We have $\phi(x)=\frac{F^{\prime \prime}(x)}{R^{\prime}(x)}=\frac{r}{a x}\left(2 x^{3}-2 a x^{2}+a^{2} x-a^{2} K\right)$ and $\phi^{\prime}(x)=\frac{r}{a x^{2}} f(x)$, where $f(x)=4 x^{3}-2 a x^{2}+a^{2} K$. The minimum value of $f(x)$ is obtained at $x_{0}=a / 3$ with $f(a / 3)=a^{2}(K-2 a / 27)$. Since $K>(3+2 \sqrt{2}) a>2 a / 27$, $f(x)>0$ for all $x>0$. The result then follows from Corollary 1 .

Next we let $F^{\prime}\left(x^{*}\right)>0$. Again the existence of a limit cycle is guaranteed by the Poincaré-Bendixson theorem because $\left(x^{*}, y^{*}\right)$ is a repeller. If there is a unique limit cycle, then it has to be a global attractor because otherwise, a second limit cycle must exist.

Consider the function $G_{\alpha}(x):=G(\alpha, x)=F^{\prime}(x)+\alpha(c-d R(x))$. For any real $\alpha$, we have $G_{\alpha}(x) \rightarrow-\infty$ as $x \rightarrow 0^{+}$or as $x \rightarrow+\infty$, and $G_{\alpha}\left(x^{*}\right)>0$. This implies that $G_{\alpha}(x)$ has (at least) two roots $x_{1}, x_{2}$ with $0<x_{1}<x^{*}<x_{2}$. We can assume that $x_{1}$ is the largest root less than $x^{*}$ and $x_{2}$ the smallest root greater than $x^{*}$. By the Mean Value Theorem, $G_{\alpha}^{\prime}(x)$ has a root $x_{3} \in\left(x_{1}, x_{2}\right)$. On the other hand, $G_{\alpha}^{\prime}(x)=0$ is equivalent to $\phi(x)+\alpha d=0$, where $\phi(x)$ is as given above. Note that $\phi\left(0^{+}\right)=-\infty, \phi(+\infty)=+\infty$, and $\phi^{\prime}(x)>0$ for $x>0$, so the equation $G_{\alpha}^{\prime}(x)=0$ has a unique solution for any given $\alpha$. It follows that $G_{\alpha}(x)$ has no positive multiple root. The uniqueness of limit cycles then follows from Lemma 1.

\section{5. $\theta$-LOGISTIC MODELS}

Next we consider the Holling Type II response function $p(x)=\frac{x}{a+x}$ with $a>0$ and the so-called $\theta$-logistic growth function $g(x)=r\left[1-\left(\frac{x}{K}\right)^{\theta}\right]$ (Goel et al. [5]). The special case when $\theta=2$ was considered by Kuang and Freedman [12, in which the authors proved the uniqueness of limit cycles. We will consider the general case $\theta>1$.

Theorem 3. Let $g(x)$ and $p(x)$ be as given above and let $\theta>1$. Then system (1) has no cycle if $F^{\prime}\left(x^{*}\right) \leq 0$. It has a unique stable limit cycle if $F^{\prime}\left(x^{*}\right)>0$.

Proof. First let $F^{\prime}\left(x^{*}\right) \leq 0$. Check that $\phi^{\prime}(x)=\frac{r \theta x^{\theta-1}}{a K^{\theta}}\left[a(\theta-1) \theta+(\theta+1)^{2} x\right]>0$ for $x>0$. Corollary 1 again implies that the system has no cycle.

Next let $F^{\prime}\left(x^{*}\right)>0$. A similar argument as in the proof for the inverted Ivlev's response function above can be made for the uniqueness of limit cycles, but we will supply a different proof because the proof is interesting. The idea is to use a criterion 
developed by Kuang and Freedman [12, which is, in our notation, $\frac{d}{d x}\left(\frac{F^{\prime}(x)}{c-d R(x)}\right) \leq 0$ for $x>0$. It can be shown that this criterion is equivalent to Lemma 1 because it was obtained by converting the system into a generalized Liénard system. See the proof of Lemma 1.4 in Kooij and Zegeling [10] for motivation.

We can rewrite $\frac{F^{\prime}(x)}{c-d R(x)}$ as $\frac{r\left[K^{\theta} x-a \theta x^{\theta}-(\theta+1) x^{\theta+1}\right]}{K^{\theta}(c-d)\left(x-x^{*}\right)}$, where $x^{*}=\frac{a d}{c-d}$. Then

$$
\frac{d}{d x}\left(\frac{F^{\prime}(x)}{c-d R(x)}\right)=\frac{r}{K^{\theta}(c-d)\left(x-x^{*}\right)^{2}} f(x),
$$

where $f(x)=-K^{\theta} x^{*}+a \theta^{2} x^{*} x^{\theta-1}+\left[(\theta+1)^{2} x^{*}-a \theta(\theta-1)\right] x^{\theta}-\theta(\theta+1) x^{\theta+1}$.

Then $f^{\prime}(x)=\theta x^{\theta-2}\left(x^{*}-x\right)\left[a \theta(\theta-1)+(\theta+1)^{2} x\right]$. Hence, the maximum of $f(x)$ is attained at $x^{*}$. But $f\left(x^{*}\right)=\frac{-K^{\theta} x^{*}}{r} F^{\prime}\left(x^{*}\right)<0$. Thus the condition for uniqueness is satisfied.

\section{OTher MODELS}

We have seen that for a variety of Gause-type models, a sufficient and necessary condition for the nonexistence of cycles is that the positive equilibrium $\left(x^{*}, y^{*}\right)$ lies on a downslope of the prey isocline $F(x)$. This adds more credit to the so-called paradox of enrichment proposed by Rosenzweig [15. This is also supported by some other recent work on system (1) with the logistic growth function $g(x)=r(K-x)$. We mention four examples below.

First consider $p(x)=\frac{x^{2}}{a+b x+x^{2}}$ with $a, b>0$. This is a combination of Holling Types II $(a=0)$ and III $(b=0)$ response functions. Using our method it is easy to show that system (1) has no cycle if and only if $F^{\prime}\left(x^{*}\right) \leq 0$. This is exactly what was obtained in Lindström [13, with different notations and terminologies used. Although the result is not new, our method is much simpler.

For the generalized Holling type response functions $p(x)=\frac{x^{n}}{a+x^{n}}$ with $n, a>0$, Sugie et al. [19] and Sugie and Katayama [18] proved that system (1) has no cycle if and only if

$$
[n d-(n-2) c] x^{*} \geq K[n d-(n-1) c] .
$$

One can check that this condition is equivalent to $F^{\prime}\left(x^{*}\right) \leq 0$.

For Ivlev's response function $p(x)=1-e^{-a x}$ with $a>0$, the necessary and sufficient condition given in Sugie [17] (for the special case $K=c=1$, which is equivalent to the general case with some rescaling) for the nonexistence of cycles is

$$
a \leq-\frac{2 d+(1-d) \ln (1-d)}{d+(1-d) \ln (1-d)} \ln (1-d) .
$$

This impossible condition does not seem to conform to any geometrical interpretation, but we can show that it again means exactly what one would suspect: $F^{\prime}\left(x^{*}\right) \leq 0$.

Under some general conditions on the response function $p(x)$, Moghadas [14] showed that (for the special case $K=1$ ) system (1) has no cycle if and only if

$$
2 x^{*}+x^{*}\left(1-x^{*}\right) \frac{p^{\prime}\left(x^{*}\right)}{p\left(x^{*}\right)} \geq 1 \text {. }
$$

Once more, this means that $F^{\prime}\left(x^{*}\right) \leq 0$. Note that the inequality given in Moghadas 14 is strict, which is a mistake. 
In addition to the examples studied in this paper, our method can be applied to other models to develop new nonexistence results. We have assumed a relatively simple form for system (1) so that our results can be easily interpreted and more readily applied. The results in Sections 2 and 3 can be extended to more general Gause-type models.

\section{REFERENCES}

[1] Cheng, K.-S. (1981), "Uniqueness of a limit cycle for a predator-prey system," SIAM J. Math. Anal., 12, 541-548. MR0617713 (82h:34035)

[2] Cheng, K.-S., S.-B. Hsu and S.-S. Lin (1981), "Some results on global stability of a predatorprey system," J. Math. Biol., 12, 115-126. MR0631003 (83c:34069)

[3] Ding, S.-H. (1989), "On a kind of predator-prey system," SIAM J. Math. Anal., 20, 14261435. MR1019308 (91f:92018)

[4] Freedman, H. I. (1987), Deterministic Mathematical Models in Population Ecology, 2nd ed., HIFR Consulting LTD, Edmonton, Canada.

[5] Goel, N. S., S. C. Maitra and E. W. Montroll (1971), "On the Volterra and other nonlinear models of interacting populations," Rev. Mod. Phys, 43, 231-276. MR0484546 (58:4448)

[6] Hofbauer, J. and J. W. H. So (1990), "Multiple limit cycles for predator-prey models," Math. Biosci., 99, 71-75. MR1055740(91g:92016)

[7] Hsu, S. B. (1978), "On the global stability of a predator-prey system," Math. Biosci., 39, 1-10. MR0472126 (57:11837)

[8] Huang, X.-C. and S. J. Merrill (1989), "Conditions for uniqueness of limit cycles in general predator-prey systems," Math. Biosci., 96, 47-60. MR.1013301(90m:92056)

[9] Kolmogorov, A. (1936), "Sulla teoria di Volterra della lotta per l'esistenza," Gi. Inst. Ital. Attuari, 7, 74-80.

[10] Kooij, R. E. and A. Zegeling (1997), "Qualitative properties of two-dimensional predator-prey systems," Nonlin. Anal., TMA, 29, 693-715. MR.1452753

[11] Kuang, Y. (1990), "Global stability of Gause-type predator-prey systems," J. Math. Biol., 28, 463-474. MR1057049 (91g:92017)

[12] Kuang, Y. and H. I. Freedman (1988), "Uniqueness of limit cycles in Gause-type models of predator-prey systems," Math. Biosci., 88, 67-84. MR0930003 (89g:92045)

[13] Lindström, T. (1989), "A generalized uniqueness theorem for limit cycles in a predator-prey system," Acta Acad. Abo., Ser. B, 49(2), 1-9. MR.1041880 (91b:92038)

[14] Moghadas, S. M. (2002), "Some conditions for the nonexistence of limit cycles in a predatorprey system," Appl. Anal., 81(1), 51-67. MR1926801(2003i:34074)

[15] Rosenzweig, M. L. (1971), "Paradox of enrichment: destabilization of exploitation ecosystems in ecological time," Science, 171, 385-387.

[16] Ruan, S. and D. Xiao (2001), "Global analysis in a predator-prey system with nonmonotonic functional response," SIAM J. Appl. Math., 61, 1445-1472. MR1813689 (2001k:92052)

[17] Sugie, J. (1998), "Two-parameter bifurcation in a predator-prey system of Ivlev type," $J$. Math. Anal. Appl., 217, 349-371. MR1492094 (98m:92024)

[18] Sugie, J. and M. Katayama (1999), "Global asymptotic stability of a predator-prey system of Holling type," Nonlinear Analysis, 38(1), 105-121. MR1693000(2000j:92026)

[19] Sugie, J., R. Kohno and R. Miyazaki (1997), "On a predator-prey system of Holling type," Proc. Amer. Math. Soc., 125, 2041-2050. MR:1396998 (97m:92005)

[20] Wolkowicz, G. S. K. (1988), "Bifurcation analysis of a predator-prey system involving group defense," SIAM J. Appl. Math., 48, 592-605. MR0941102 (89g:92058)

[21] Xiao, D. and Z. Zhang (2003), "On the uniqueness and nonexistence of limit cycles for predator-prey systems," Nonlinearity, 16(3), 1185-1201. MR1975802 (2004c:34084)

Department of Mathematics, Pittsburg State University, Pittsburg, Kansas 66762

E-mail address: yliu@pittstate.edu 